\title{
Efeitos da prática mental na aquisição de habilidades motoras em sujeitos novatos
}

CDD. 20.ed. 152.3

\author{
Thábata Viviane Brandão GOMES* \\ Herbert UGRINOWITSCH* \\ Nádia Fernanda Schmitt MARINHO* \\ Rodolfo Novellino BENDA*
}

*Universidade Federal de Minas Gerais.

\section{Resumo}

Estudos de prática mental com sujeitos novatos apontam sua superioridade à ausência de prática. Entretanto, pré-teste ou familiarização usando prática física podem influenciar o efeito da prática mental. Este estudo investigou os efeitos da prática mental em novatos na tarefa praticada, assim como 0 posicionamento da prática física em relação à prática mental. Vinte e cinco universitários voluntários praticaram uma tarefa seriada de posicionamento e foram distribuidos em cinco grupos conforme o tipo de prática: física (GPF), mental (GPM), física-mental (GPFM), mental-fisica (GPMF), e grupo controle (GC) que participou apenas dos testes. Uma Anova não paramétrica aplicada nos testes mostrou superioridade dos grupos com prática física (GPF, GPFM e GPMF) sobre os grupos controle e prática mental. Ao considerar a pequena amostra utilizada, os resultados sugerem que a prática mental em sujeitos novatos depende da prática física para ser efetiva independente de seu posicionamento (antes ou após).

Unitermos: Prática mental; Prática física; Aprendizagem motora; Novatos.

\section{Introdução}

Um dos fatores do processo de aprendizagem motora, que tem sido regularmente investigado e considerado como o mais importante é a prática (Schmidt \& Lee, 1999). Dentre as variáveis estudadas em prática, podem ser citadas a sua variabilidade, organização, segmentação, distribuição e a prática mental (Tani, Meira Junior, Ugrinowitsch, Benda, Chiviacowsky \& Corrêa, 2010; UgrinoWITSCH \& BENDA, 2011). A prática mental pode ser entendida como a imaginação da realização de habilidades motoras sem a execução de movimentos (Adegbesan, 2009; Magill, 2007; Richardson, 1967a; Yves, 2003). Em outras palavras, a prática mental é o ensaio interno de uma habilidade motora na ausência de movimentos físicos explícitos dos segmentos corporais envolvidos (Debarnot, Creveaux, Collet, Gemignani, Massarelli, Doyon \& Guillot, 2009; Driskell, Copper \& Moran, 1994; Gentili, Han, Schweighofer \& Papaxanthis; 2010; Gentili, Papaxanthis \& Pozzo, 2006; UGRINOWITSCH \& BENDA, 2011).
Diferentes teorias procuram explicar a influência da prática mental sobre a aprendizagem motora. A teoria psiconeuromuscular postula que a prática mental facilita a aquisição de habilidades motoras reforçando uma representação motora através da ativação dos músculos que participam do movimento imaginado (Magill, 2007; SuInN, 1993). A teoria de ativação explica que no momento da prática mental, a atenção deve ser focalizada nos aspectos relevantes das tarefas e assim elimina estímulos irrelevantes que podem prejudicar o desempenho da habilidade (CorbIN, 1972). A teoria da aprendizagem simbólica propóe que a aprendizagem e a repetição dos símbolos que compóem um movimento contribuem para a aquisição de habilidades motoras (FELTZ \& LANDERS, 1983). Por fim, a teoria bioinformacional explica que a prática mental fortalece o processamento da informação desde a identificação do estímulo até a seleção e programação da resposta (SUINN, 1993).

A prática mental tem sido um procedimento recomendado para intervenção, sendo sugerida sua 
utilização em aulas de educação física e na iniciação esportiva, com o objetivo de aprender uma nova habilidade motora (BARELA \& ISAYAMA, 1995; BROUZIYNE \& MolinARO, 2005; UGRINOWITSCH \& BENDA, 2011), para o constante aperfeiçoamento técnico com atletas no treinamento esportivo (ADEGBESAN, 2009; Fontani, Migliorini, Benocci, Facchini, Casini \& CorradesChi, 2007; SHOEKFELT \& GRIFFITH, 2008), como também para a reabilitação motora (Allami, Paulignan, Brovelli \& Boussaoud, 2008; Maring, 1990; RoosinK \& ZIJDEWIND, 2010). Entretanto, tais recomendações para sua utilização na intervenção têm sido constantemente aprimoradas, visto que pesquisas sobre o tema têm encontrado novos subsídios sobre como fatores estudados em prática mental podem contribuir na aprendizagem motora.

Os temas investigados em prática mental têm sido amplos e envolvem muitas vezes questôes similares já estudadas em prática física. Dentre os fatores estudados, podem ser citados a duração das sessões ou quantidade de prática (ETNIER \& LANDERS, 1996; Feltz \& Landers, 1983; Gomes, Ugrinowitsch, Coelho, Marinho, Fonseca \& Benda, 2012; MaCKAY, 1981; Richardson, 1967a; WeInBERG, HANKES \& JACKSON, 1991), nível de habilidade dos sujeitos na tarefa (BEILOCK \& Gonso, 2008; Harris \& RoBinson, 1986; NaITo, Kato \& FuKUdA, 2004), tipo de tarefa - habilidades abertas ou fechadas (CoelHo, Campos, Silva, Okazaki \& Keller, 2007; Feltz \& Landers, 1983; McBrjde \& Rothstein, 1979); demanda motora ou cognitiva (FeLTZ \& LANDERS, 1983; Hird, Landers, Thomas \& Horan, 1991; RYAN \& SimONS, 1981, 1983), prática mental na perspectiva interna ou externa (EPSTEIN, 1980; HARRIS \& Robinson, 1986; Roosink \& Zijdewind, 2010) e combinações entre prática física e mental (ETNIER \& LANDERS, 1996; Gomes et al., 2012; Millard, MaHONEY \& WardRop, 2001; RAISBECK, WyatT \& SheA, 2012; Stebbins, 1968; Weinberg, Hankes \& JACKSON, 1991). Contudo, novas questōes são levantadas mesmo considerando o extenso conhecimento já produzido sobre o tema. Como novos moderadores (fatores que influenciam os resultados da variável), por exemplo, pesquisas verificaram a persistência dos efeitos da prática mental após uma noite de sono (DEBARNOT et al., 2009), a combinação de prática mental e estruturação de prática (OvERDORF, SCHWEIGHARDT, Page \& McGrath, 2004) ou a combinação entre prática mental e demonstração (RAm, RigGS, SKaling, LANDERS \& MCCullagh, 2007).

Apesar dos fatores mencionados anteriormente, uma parcela dos estudos ainda busca compreender como a prática mental promove aprendizagem motora e para isso utilizam delineamento experimental constituído por grupo controle e grupos de prática mental, física ou combinada (física e mental) (Brouziyne \& Molinaro, 2005; Fontani et al., 2007; Gentili, Papaxanthis \& Pozzo, 2006; Gentile et al., 2010; Gomes, Ugrinowitsch \& Benda, 2009; Magill, 2007; RaisbeCK, Wyatt \& SHEA, 2012). Dentre os resultados encontrados, as pesquisas têm sido consistentes em mostrar que a prática física é superior à prática mental e esta, por sua vez, superior à ausência de prática (Allami et al., 2008; Denis, 1985; Driskell, Copper \& Moran, 1994; Feltz \& Landers, 1983; Grouios, 1992; Millard, Mahoney \& Wardrop, 2001; RicharDSON, 1967a, 1967b). Ao analisar os resultados da prática combinada, observa-se a sua similaridade com a prática física e consequente superioridade sobre a prática mental (BARELA \& ISAYAMA, 1995; Hirai \& GobBI, 1990; MacKay, 1981; RichardSON, 1967a) ou mesmo sua superioridade sobre a própria prática física (Allami et al., 2008; Maring, 1990; McBrjde \& Rothstein, 1979; Stebisns, 1968).

A utilização de grupos de prática combinada para investigar a prática mental tem sido uma interessante estratégia que apresentou novos resultados e interpretações. Mais do que verificar se a prática mental promove ou não a aprendizagem, como no caso de um delineamento experimental que compara um grupo de prática mental com um grupo controle, a utilização da prática combinada em delineamento com três grupos experimentais permite verificar como ou quanto a prática mental influencia a aprendizagem (MAgill, 2007). Como exemplo, há os resultados supracitados em que a prática mental é inferior à prática física e combinada. Ao analisar a prática mental isolada, este resultado seria desfavorável para sua utilização. Contudo, o benefício da prática mental tem sido ressaltado ao considerar a similaridade entre prática física e prática combinada. A prática combinada tem sido investigada com composição de $50 \%$ de prática física e $50 \%$ de prática mental, organizada em alguns estudos por blocos (BARELA \& Isayama, 1995; Millard, Mahoney \& Wardrop, 2001; Raisbeck, Wyatt \& Shea, 2012; Stebbins, 1968) e em outros de forma alternada (MCBRJDE \& RothSTEIN, 1979). Conforme o conjunto de resultados encontrados, é possível concluir que a prática combinada tem uma participação importante da prática mental, visto que os sujeitos realizam uma quantidade menor de prática física comparada ao grupo especificamente de prática física. 
A partir destes resultados, verificam-se na literatura duas novas facetas de investigação da prática combinada: diferentes proporçōes de prática física e prática mental (outras proporçôes além de 50\% - 50\%) e a ordem de apresentação da prática mental (antes ou após a prática física). Estudos sobre diferentes proporçōes de prática física e mental não chegaram a um consenso sobre qual a melhor proporção. Em um estudo (Allami et al., 2008), as proporçôes mais altas de prática mental foram mais efetivas, mas em outros dois estudos (CAHN, 2008; HIRD et al., 1991) os resultados não mostraram diferença entre as proporções mais altas ou mais baixas de prática mental. Do mesmo modo, a ordem de apresentação da prática mental tem encontrado superioridade da prática mental antes da física (ETNIER \& Landers, 1996; Raisbeck, Wyatt \& Shea, 2012), assim como similaridade entre as condiçôes (GOMES et al., 2012; WeinberG, Hankes \& JaCKSON, 1991). Como consequência destes resultados, o problema de investigação do presente estudo foi levantado.

Além da observada efetividade da proporção de $50 \%$ de prática física na prática combinada, novos achados têm destacado que não se consegue aprender uma habilidade completamente nova apenas com prática mental (Mulder, Zijlstra, Zijlstra \& HoCHSTENBACH, 2004). Além disso, conforme Allami et al. (2008), apenas $25 \%$ de prática física na prática combinada já foram suficientes para resultar em superioridade sobre $100 \%$ de prática física. É possível que a quantidade de prática física, quando utilizada em prática combinada, necessária para promover aprendizagem não seja alta. Isto é, uma pequena experiência anterior com a tarefa, por menor que seja, pode ser suficiente para resultar em aproveitamento distinto da prática mental. Por exemplo, o estudo de Mulder et al. (2004) demonstrou que houve superioridade na aprendizagem quando a prática mental foi aplicada a sujeitos que já conseguiam iniciar o movimento (denominado grupo "já iniciando a execução") do que quando não realizavam o movimento (denominado grupo "zero absoluto").

É comum observar nos estudos tradicionais de prática mental o uso de prática física prévia à fase de aquisição por meio de pré-teste (BrouZIYNE \& Molinaro, 2005; Coelho et al., 2007; Hird et al., 1991; ZECKER, 1982) ou de familiarização à tarefa do experimento (AlLAmi et al., 2008; BrouZIYNE \& Molinaro, 2005; Millard, Mahoney \& Wardrop, 2001; Overdorf et al., 2004; RaISBECK, WyATt \& SHEA, 2012; RyAn \& SimONS, 1983). Estes estudos não mencionam na descrição do método os critérios para definir o número de tentativas de prática física. Tais critérios deveriam estabelecer para a prática combinada um número de tentativas de prática física insuficiente para a redução do erro, de forma a identificar o papel da prática mental. De modo contrário, a prática física assumiria a responsabilidade exclusiva da aprendizagem.

Em síntese, a prática mental aplicada a novatos é mais efetiva que a ausência de prática e é menos efetiva que a prática física. Quando combinada à prática física, seu efeito é superior à prática mental isolada e similar à prática física. Ao considerar que procedimentos metodológicos como pré-teste ou familiarização em prática física foram utilizados nos estudos sobre prática mental, é possível esperar alguma influência destes procedimentos nos efeitos da prática mental. Seria então necessário conduzir um estudo em que a prática mental fosse testada sem qualquer influência de prática física. Ao assumir que a prática física tem um importante papel para a efetividade da prática mental, o presente estudo levanta a seguinte questão: a prática mental, sem qualquer experiência física, é efetiva para a aprendizagem motora? Assim, o objetivo do presente estudo foi investigar os efeitos da prática mental na aquisição de habilidades motoras em sujeitos novatos. Ao considerar que a prática física é essencial para aquisição de habilidades motoras em sujeitos novatos, esperase que os grupos com prática física sejam superiores aos grupos de prática mental e controle, que seriam similares entre si. Em relação aos grupos de prática combinada, dos quais se espera um resultado similar ao grupo de prática física, procurou-se investigar também a ordem de apresentação da prática mental (antes ou após a prática física).

\section{Método}

\section{Amostra}

O estudo foi composto por 25 universitários voluntários, que se autodeclararam destros, com idade
$\mathrm{M}=24,28$ anos, $\mathrm{DP}=3,61$ anos, sendo 15 homens e 10 mulheres, novatos na tarefa, sem experiência com algum tipo de prática mental e tarefas de controle de tempo. O estudo foi previamente aprovado 
pelo Comitê de Ética e Pesquisa (ETIC 213/08), e os voluntários autorizaram sua participação ao assinar o termo de consentimento livre e esclarecido.

\section{Instrumento e tarefa}

Foi utilizado um aparelho composto por uma plataforma de madeira e uma central de controle ligada a um microcomputador. A plataforma de madeira (107 cm de largura; $64 \mathrm{~cm}$ de comprimento; e $10 \mathrm{~cm}$ de altura) continha seis recipientes $(12 \mathrm{~cm}$ de diâmetro; $5 \mathrm{~cm}$ de profundidade) enumerados de um a seis distribuídos em duas linhas (1 a 3 mais próxima e 4 a 6 mais distante da posição do voluntário). A central de controle continha um conjunto de diodos (LEDs) responsáveis por prover o estímulo visual para início da tarefa e uma chave de respostas que deveria ser solta no início e pressionada após a execução, quando a tentativa era finalizada (FIGURA 1). Um "software" para medir o tempo de resposta e armazenar os dados também foi utilizado.

A tarefa constituiu no transporte de bolas de tênis (posicionamento manual) em sequenciamento e tempo alvo pré-determinados utilizando a mão não preferencial (como todos os voluntários se autodeclararam destros, foi utilizada a mão esquerda). A tarefa envolveu precisão espacial (acertar a sequência) assim como precisão temporal (execução no tempo estabelecido).

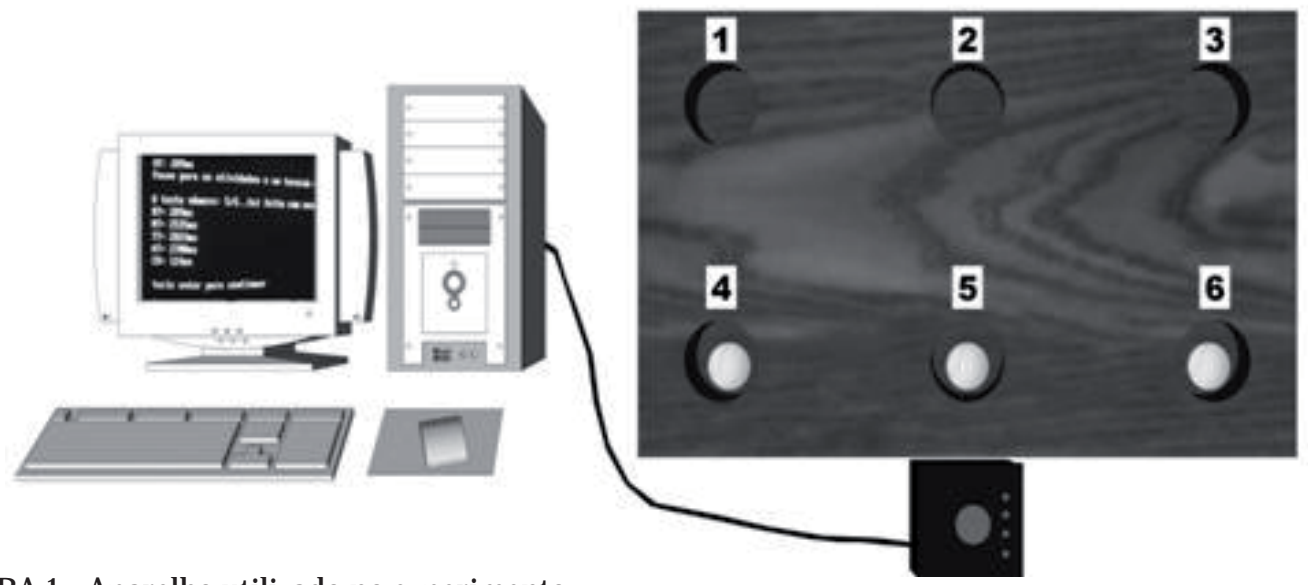

FIGURA 1 - Aparelho utilizado no experimento.

\section{Delineamento experimental}

Os sujeitos foram aleatoriamente distribuídos em quatro grupos experimentais e um grupo controle $(\mathrm{n}=5)$ : prática física $(\mathrm{GPF})$, prática mental $(\mathrm{GPM})$, prática física e mental (GPFM), prática mental e física (GPMF) e grupo controle (GC). A distribuição resultou na seguinte composição: GPM (quatro homens e uma mulher com idade $\mathrm{M}=24,4$ anos; $\mathrm{DP}=1,95$ anos); GPF (dois homens e três mulheres com idade $\mathrm{M}=24,4$ anos, $\mathrm{DP}=2,97$ anos); $\mathrm{GPFM}$ (três homens e duas mulheres com idade $\mathrm{M}=26,2$ anos, DP $=5,93$ ); GPMF (três homens e duas mulheres com idade $\mathrm{M}=23,4$ anos, $\mathrm{DP}=4,28$ anos); e GC (três homens e duas mulheres com idade M $=23$ anos, $\mathrm{DP}=2,12$ anos).

$\mathrm{Na}$ fase de aquisição, os sujeitos praticaram 10 tentativas na sequência 5-1/4-5/6-4 (transportar a bola do recipiente 5 para o recipiente 1 ; do recipiente 4 para o 5; e do recipiente 6 para o 4), com o tempo alvo de $3500 \mathrm{~ms}$, conforme as características de cada grupo. Os grupos de prática combinada (GPFM e GPMF) realizaram cinco tentativas em cada condição (física e mental). Os sujeitos receberam conhecimento de resultados (diferença entre tempo gasto e tempo alvo) após cada tentativa de prática física da fase de aquisição. Os sujeitos do grupo controle não participaram da fase de aquisição, apenas dos testes. O teste de retenção foi aplicado após intervalo de 24 horas em todos os grupos e manteve as mesmas exigências da fase de aquisição com 10 tentativas de prática física. Cinco minutos após o teste de retenção, foi realizado o teste de transferência com 10 tentativas de prática física com um novo sequenciamento 5-3/6-5/4-6 (transportar a bola do recipiente 5 para o recipiente 3 ; do recipiente 6 para o 5 ; e do recipiente 4 para o 6) e tempo alvo de 4500 ms. Não foi fornecido conhecimento de resultados durante os testes.

Dois estudos piloto foram realizados para a definição da tarefa, com vistas a controlar a quantidade de prática para que $50 \%$ de prática física fosse insuficiente para a redução do erro sem uso de pré-teste ou familiarização. O primeiro teve como objetivo comparar o desempenho utilizando a mão preferencial e a mão não preferencial com o 
então novo sequenciamento (5-1/4-5/6-4) e tempo alvo (3500 ms) com resultados já encontrados em estudos prévios, que utilizaram a mesma tarefa com sequenciamento 4-2/5-3/6-1 e tempo alvo de 2700 ms (Gomes \& Benda, 2005) e de 3000 ms (Vieira, UGRINOWITSCH, LAGE \& BENDA, 2011) para a fase de aquisição. $O$ primeiro estudo piloto mostrou ser a condição mão não preferencial, nova sequência e novo tempo alvo mais difícil, pois resultou em pior desempenho daqueles observados nos estudos citados. $\mathrm{O}$ primeiro estudo piloto ainda verificou que o tempo mais longo no teste de transferência (4500 ms) apresentou pior desempenho do que um tempo mais curto (2500 ms). Assim, esta condição foi a escolhida por requerer maior exigência no teste.

$\mathrm{O}$ segundo estudo piloto analisou o número de tentativas buscando verificar o momento em que houve uma primeira redução do erro em relação ao desempenho apresentado na primeira tentativa. Os resultados apontaram para uma redução do erro a partir da sexta tentativa. Seria até plausível pensar que se houvesse continuidade da prática, o erro pudesse ainda ser reduzido. Entretanto, conforme o desenho experimental proposto neste estudo, a metade de tentativas de prática física não poderia ser suficiente para conduzir à redução do erro. Esta condição foi adotada como um modo de controle do número de tentativas de prática física nos grupos de prática combinada. Assim, uma possível redução do erro nos grupos de prática combinada seria fruto de contribuição efetiva da prática mental. Com isso, decidiu-se por utilizar 10 tentativas de prática na fase de aquisição.

\section{Procedimentos}

Os participantes posicionaram-se em pé em frente ao aparelho e receberam instrução verbal e demonstração sobre a tarefa. Ao comando verbal "prepara", os voluntários deveriam pressionar a chave de resposta e, ao acendimento dos diodos (sinal visual para iniciar a tarefa), deveriam soltar a chave de resposta e realizar o transporte das três bolas de tênis de acordo com a sequência e tempo alvo estabelecidos, finalizando a tentativa com um novo acionamento da chave de resposta. $\mathrm{Na}$ condição de prática mental, ao comando verbal "prepara”, os sujeitos deveriam fechar os olhos. Em seguida, deveriam sinalizar com a mão não preferencial quando fossem iniciar a imaginação da tarefa (imaginação do acendimento dos diodos); imaginariam toda a sequência da tarefa sentindo a execução do movimento, no tempo estabelecido, na ausência de movimento observável e, ao término da tentativa (imaginação da mão não preferencial pressionando a chave de resposta), os voluntários sinalizariam novamente com a mesma mão e abririam os olhos. Com isso, não foi mensurado o tempo de cada tentativa durante a prática mental. $\mathrm{O}$ experimentador controlou o número de tentativas através da sinalização do voluntário com a mão.

\section{Análise dos dados}

Os dados foram organizados em blocos de cinco tentativas, resultando em dois blocos na fase de aquisição (A1 e A2), dois blocos de teste de retenção (R1 e R2) e dois blocos de testes de transferência (T1 e T2). Os efeitos das variáveis dependentes foram analisados por meio da precisão do desempenho (erro absoluto - diferença de tempo entre a meta e o tempo realizado) e consistência do desempenho (desvio padrão do erro absoluto). Nos últimos 10 anos, alguns estudos que investigaram a prática mental utilizaram como medida de precisão a raiz quadrada do erro médio (Gentili et al., 2010; TOUSAINT \& BLANDin, 2010) ou o erro absoluto (Debarnot et al., 2009; Yves, 2003). Considerando que a presente tarefa envolve a precisão temporal, a escolha do erro absoluto para sua mensuração parece adequada. Assim, para analisar a consistência deste erro, foi calculado o seu desvio padrão.

Com uma amostra de cinco sujeitos por grupo e, pela falta de normalidade e homogeneidade de variância dos dados (analisados respectivamente pelo teste de Shapiro-Wilk e pelo teste de Levene), foram utilizados testes não paramétricos. $\mathrm{Na}$ fase de aquisição foi utilizado o teste de Wilcoxon para análises intra-grupo e o teste de Mann-Whitney para análises inter-grupos. A Anova não paramétrica (BRunner, Domhof \& LANGer, 2002) em esquema fatorial (cinco grupos $\mathrm{x}$ dois blocos) foi utilizada para o teste de retenção e para os teste de transferência, sendo adotado o teste de Dunn como "post-hoc". 


\section{Resultados}

Na medida de erro absoluto, na fase de aquisição, a análise intra-grupo identificou diferença significativa no GPF, indicando redução do erro entre o primeiro e segundo bloco de tentativas $[Z(n=5)=2,02$, $p=0,043$ ] (FIGURA 2). Não foi identificada mudança no comportamento do GPFM e GPMF. A comparação entre os grupos não verificou diferença significante no primeiro bloco de tentativas da fase de aquisição entre os grupos GPF e GPFM [Z(n $=5)=-0,31, p=0,75]$, entretanto, foi observado menor erro do GPF em relação ao GPMF [Z(n = 5) $=-1,98, p=0,047]$.
Nos testes de retenção (FIGURA 2), a análise do erro absoluto não encontrou diferença significante entre blocos $[\mathrm{H}(1)=1,04, \mathrm{p}=0,31]$ ou interação entre grupos e blocos $[\mathrm{H}(4)=1,53, \mathrm{p}=0,82]$. Observou-se diferença significativa no fator grupos $[\mathrm{H}(4)=26,63$, $\mathrm{p}<0,0001]$ e o teste "post-hoc" de Dunn registrou melhor desempenho dos grupos GPF, GPFM e GPMF sobre os grupos GPM e GC $(p<0,05)$. Nos testes de transferência não foi encontrada diferença significante entre grupos $[\mathrm{H}(4)=8, \mathrm{p}=0,091]$, entre blocos $[\mathrm{H}(1)=0,56, \mathrm{p}=0,455]$ ou interação entre grupos e blocos $[\mathrm{H}(4)=0,58, \mathrm{p}=0,966]$.

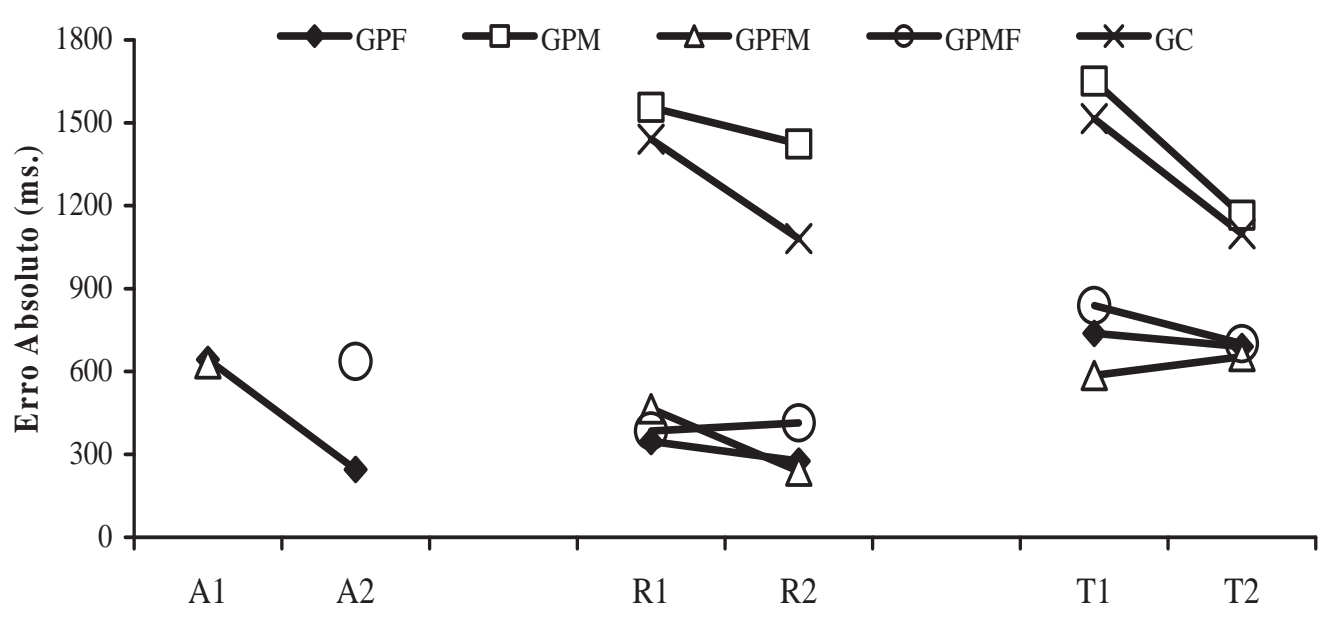

Blocos de 5 tentativas na fase de aquisição (A1 e A2) e nos testes de retenção (R1 e R2) e de transferência (T1 e T2)

FIGURA 2 - Média do Erro Absoluto em blocos de cinco tentativas na fase de aquisição, testes de retenção e de transferência.

Na medida do desvio padrão do erro absoluto, na fase de aquisição, não foi registrada diferença significante no GPF entre o primeiro e segundo bloco de tentativas $[\mathrm{Z}(\mathrm{n}=5)=1,75, \mathrm{p}=0,079]$ (FIGURA 3). Na comparação inter-grupos também não verificou diferença significante no primeiro bloco de tentativas da fase de aquisição entre os grupos GPF e GPFM [Z $(n=5)=-0,1, p=0,91]$, entretanto, foi observada menor variabilidade do GPF em relação ao GPMF $[\mathrm{Z}(\mathrm{n}=5)=-2,19, \mathrm{p}=0,028]$.
Nos testes de retenção (FIGURA 3) foi encontrada diferença significativa entre grupos $[\mathrm{H}(4)=12,66, \mathrm{p}=$ $0,013]$ e entre blocos $[H(1)=6,81, p=0,009]$. O teste "post-hoc" de Dunn indicou maior variabilidade do GPM e do GC $(p<0,05)$ em relação aos demais grupos e, para blocos, registrou que R1 foi mais variável que $\mathrm{R} 2(\mathrm{p}<0,05)$. Não foi detectada interação significante entre grupos e blocos $[\mathrm{H}(4)=1,92, \mathrm{p}=0,75]$. Nos testes de transferência e não foi encontrada diferença significante entre grupos $[\mathrm{H}(4)=8,07, \mathrm{p}=0,089]$, entre blocos $[\mathrm{H}(1)=1,93, \mathrm{p}=0,165]$ ou interação entre grupos e blocos $[\mathrm{H}(4)=2,8, \mathrm{p}=0,592]$. 


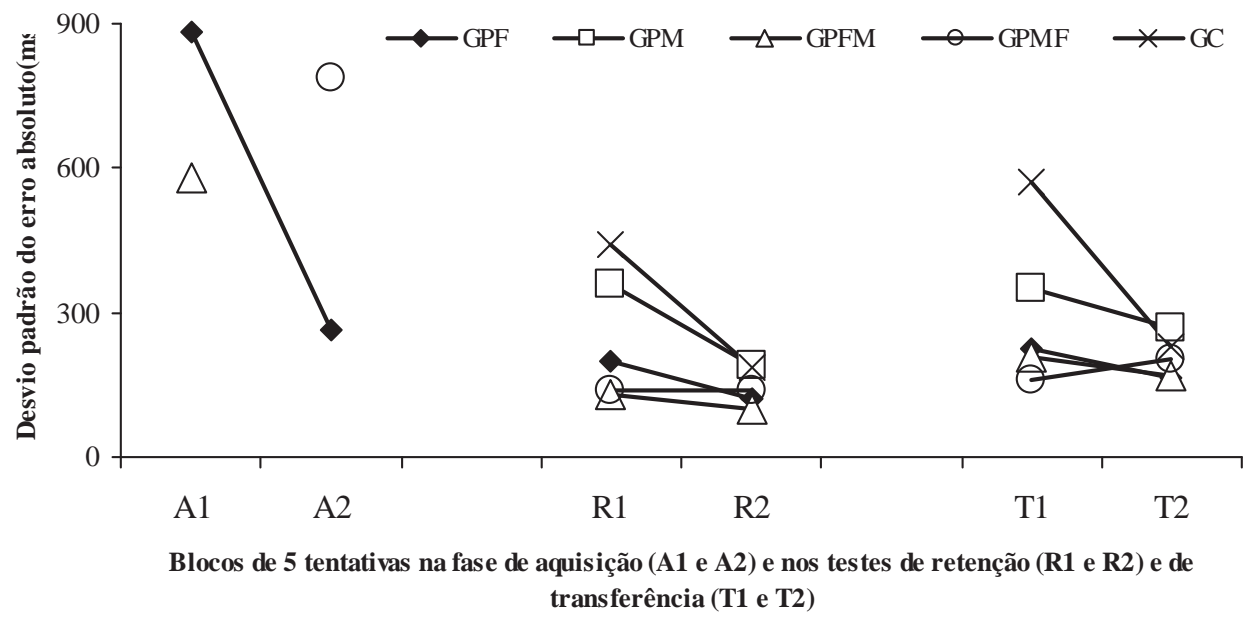

FIGURA 3 - Média do Desvio Padrão do Erro Absoluto em blocos de cinco tentativas na fase de aquisição, testes de retenção e de transferência.

\section{Discussão}

O presente estudo teve como objetivo investigar os efeitos da prática mental na aquisição de habilidades motoras em sujeitos novatos. Para tal, considerou o sujeito novato como intacto na tarefa utilizada. Cinco grupos foram formados (GPF, GPM, GPFM, GPMF e GC) e o resultado esperado seria a superioridade dos grupos que continham prática física em seu delineamento.

Os achados confirmam a hipótese apresentada, pois se verificou melhor desempenho nos testes dos grupos de prática física e combinada, ressaltando a importância da prática física para a aprendizagem motora de sujeitos novatos. Assim, os achados do presente estudo podem contribuir para lançar novos questionamentos a serem investigados, de forma a testar a assunção de que a prática mental oferece condições para a aprendizagem motora com sujeitos novatos (DENIS, 1985; Feltz \& Landers, 1983; Magill, 2007; Millard, Mahoney \& Wardrop, 2001; Suinn, 1993). Se por um lado, os resultados não demonstraram efeitos benéficos para a prática mental isolada, por outro lado, eles reforçam a eficiência da prática mental, quando combinada à prática física, o que tem sido regularmente verificado em outros estudos (Allami et al., 2008; Barela \& IsAyama, 1995; CAHN, 2008; Hirai \& GobBi, 1990; MacKay, 1981; Maring, 1990; McBrjde \& Rothstein, 1979; Raisbeck, Wyatt \& SHEA, 2012; RichardSON, 1967a; StebBins, 1968).

Esta eficiência da prática mental na prática combinada merece destaque, visto que o presente estudo controlou o número de tentativas de prática física executadas nos grupos de prática combinada, que eram insuficientes para a redução de erro. Investigar esta questão exigiu um número reduzido de tentativas na fase de aquisição, diferente dos estudos tradicionais de aprendizagem motora. Portanto, o fato de os grupos de prática combinada terem mostrado mesmo desempenho do grupo de prática física nos testes leva a reforçar o papel positivo da prática mental na aprendizagem motora.

Outro fator que ressalta o benefício da prática mental na aprendizagem motora também está relacionado ao controle efetuado neste estudo. Pesquisas que têm testado a prática mental têm utilizado tentativas de prática física antes da fase de aquisição, seja em pré-teste (Brouziyne \& Molinaro, 2005; Hird et al., 1991; Smith \& Collins, 2004; Stebbins, 1968; ZECKER, 1982) ou em familiarização à tarefa (ALLAMI et al., 2008; BrouZIYNE \& Molinaro, 2005; Millard, MAHONEY \& WARDROP, 2001; OVERDORF et al., 2004; Raisbeck, Wyatt \& Shea, 2012; Ryan \& Simons, 1983; Wohldmann, Healy \& Bourne, 2007, 2008).

No presente estudo tais procedimentos não foram adotados, pois poderiam limitar o real efeito da prática mental. Mesmo uma experiência limitada de executar a habilidade poderia ser utilizada para auxiliar a prática mental, como foi observado em MULDER et al. (2004). Tal possibilidade já havia sido sugerida em outros estudos (Harris \& RoBINSON, 1986; WeINBERG, 1982), em que uma quantidade mínima de experiência na execução da tarefa parece ser essencial para a efetividade da imaginação, isto é, a experiência prévia na tarefa 
parece influenciar os efeitos da prática mental. Chama atenção também a crítica elaborada por RYAN (1983), em que o efeito da prática mental pode ser ofuscado por causa do número de tentativas de prática física da tarefa no pré-teste. Como contraponto, em estudos que não apresentaram nem pré-teste, nem familiarização à tarefa, a prática mental não foi diferente da ausência de prática em grupo controle (GOMES, UGRINOWITSCH $\&$ BENDA, 2009; RAM et al., 2007).

A prática física parece então essencial, pois está relacionada à percepção que o sujeito tem sobre o seu desempenho. Ao executar (fisicamente) a habilidade, o sujeito obtém informações sobre a realização da tarefa que somente a prática física permite por meio da interação percepção-ação (TURVEY, 1977, 2007). Turvey (2007) destaca que a sinergia, como uma organização para o controle de vários graus de liberdade músculo-esqueléticos, é simultaneamente e necessariamente uma organização de aferência, de percepção. Isto é, para a execução do movimento coordenado, é essencial perceber este movimento. No caso da prática mental, esta aferência não ocorre, resultando em prejuízos para a aprendizagem motora.

Quanto ao posicionamento da prática mental na prática combinada (antes ou após a prática física), poucos estudos têm discutido a questão. Dentre eles há resultados em que o posicionamento é indiferente (GOMES et al., 2012; WeInBERG, Hankes \& JACKSON, 1991) e estudos em que o posicionamento anterior da prática mental foi mais efetivo (ETNIER \& LANDERs, 1996; RaISBECK, WyatT \& SCHEA, 2012).
Quando a prática mental antecede à prática física, o aprendiz é levado a superar a dificuldade de extrair as informações relevantes da tarefa, resultando em maior esforço cognitivo do que quando a prática mental sucede à prática física (ETNIER \& LANDERS, 1996). Apesar desta explanação, os resultados do presente estudo demonstram similaridades entre as condições, reforçando a noção de que o posicionamento da prática mental não é importante (GOMES et al., 2012; Weinberg, Hankes \& JaCKSON, 1991).

$\mathrm{O}$ presente estudo levantou novas questões e assumiu posições ousadas, contrárias àquelas que a literatura vem tradicionalmente apontando (FELTZ \& LANDERS, 1983; Magill, 2007). Os resultados encontrados suportaram a hipótese proposta. Assim, uma conclusão de que a prática física é importante para a aquisição de habilidades motoras poderia também ser assumida. Tal assunção não implicaria abandonar a prática mental, mas sim associá-la à prática física especialmente ao se lidar com sujeitos novatos.

Contudo, é preciso certa cautela com a generalização dos achados revelados por este estudo. A interpretação dos resultados deve considerar o tamanho da amostra, que é considerado pequeno no campo da Aprendizagem Motora. Como sugestão para futuros estudos, além da ampliação do tamanho da amostra, o que permitiria uma distribuição normal dos dados e, por conseguinte, a utilização de análise estatística paramétrica, a busca por uma tarefa que requeira maiores exigências de controle dos graus de liberdade para a sua redução do erro.

\section{Abstract}

Effects of mental practice in motor skills acquisition in novice subjects

Studies of mental practice with novices pointed out its superiority when compared to no practice at all. However, pre-test as well as familiarization using physical practice can influence the effect from mental practice. This study investigated the effects of mental practice in novice in the practiced task, as well as the location of physical practice in relation to mental practice. Twenty five undergraduate volunteers performed a serial positioning task and they were distributed in five groups in accordance to the type of practice: Physical (GPF), mental (GPM), physical-mental (GPFM), mental-physical (GPMF), and control group (GC) which took part only in tests. A non parametrical Anova applied in tests showed superiority of groups with physical practice (GPF, GPFM, and GPMF) to control and mental practice groups. In considering the small sample, the results suggest that mental practice in novice subjects depends on physical practice to be effective, regardless its location (before or after).

UnITERMs: Mental practice; Physical practice; Motor learning; Novice. 


\section{Resumen}

Efectos de la práctica mental en la adquisición de habilidades motoras en novatos

Estudios de práctica mental con sujetos sin experiencia indican su superioridad en comparación a la ausencia de esa la práctica. Entre tanto, pre-test y familiarización usando práctica física pueden influenciar el efecto de la práctica mental. Este estudio investigó los efectos de la práctica mental en novatos en la tarea practicada, bien como el posicionamiento de la práctica física en relación a la práctica mental. Veinticinco universitarios voluntarios practicaron una tarea seriada de posicionamiento y fueron distribuidos en cinco grupos conforme el tipo de práctica: fisica (GPF), mental (GPM), física-mental (GPFM), grupo mental física (GPMF) y grupo de control (GC) que participó solamente de los testes. Una Anova no paramétrica aplicada en los testes mostró la superioridad de los grupos conforme el tipo de práctica fisica (GPF, GPFM y GPMF) sobre los grupos de control y de práctica mental. Al considerar que la muestra utilizada fue pequeña, los resultados sugieren que la práctica mental en sujetos novatos depende de la práctica física para ser efectiva, independiente de su posicionamiento (antes o después).

Palabras clave: Práctica mental; Práctica física; Aprendizaje motor; Novatos.

\section{Referências}

ADEGBESAN, O.A. Use of imagery by athletes in Nigeria. Perceptual and Motor Skills, Missoula, v.108, p.43-50, 2009. ALLAMI, N.; PAULIGNAN, Y.; BROVELLI, A.; BOUSSAOUD, D. Visuo-motor learning with combination of diVerent rates of motor imagery and physical practice. Experimental Brain Research, Berlin, v.184, p.105-13, 2008.

BARELA, J.A.; ISAYAMA, H.F. Efeitos do tipo de prática na aprendizagem do estilo borboleta na natação. Movimento, Porto Alegre, v.2, p.38-45, 1995.

BEILOCK, S.L.; GONSO, S. Putting in the mind versus putting on the green: Expertise, performance time, and the linking of imagery and action. The Quarterly Journal of Experimental Psychology, London, v.61, p.920-32, 2008.

BROUZIYNE, M.; MOLINARO, C. Mental imagery combined with physical practice of approach shots for golf beginners. Perceptual and Motor Skills, Missoula, v.101, p.203-11, 2005.

BRUNNER, E.; DOMHOF, S.; LANGER, F. Nonparametric analysis of longitudinal data in factorial experiments. Hoboken: John Wiley, 2002.

CAHN, D. The effects of varying ratios of physical and mental practice, and task difficulty on performance of a tonal pattern. Psychology of Music, Thousand Oaks, v.36, p.179-91, 2008.

COELHO, R.W.; CAMPOS, W.; SILVA, S.G.; OKAZAKI, F.H.A.; KELLER, B. Imagery intervention in open and closed tennis motor skill performance. Perceptual and Motor Skills, Missoula, v.105, p.458-68, 2007.

CORBIN, C.B. Mental practice. In: MORGAN, W.P. Ergogenic aids and muscular performance. New York: Academic Press, 1972. p.94-118.

DEBARNOT, U.; CREVEAUX, T.; COLLET, C.; GEMIGNANI, A.; MASSARELLI, R.; DOYON, J.; GUILLOT, A. Sleep-related improvements in motor learning following mental practice. Brain and Cognition, Amsterdam, v.69, p.398-405, 2009.

DENIS, M. Visual imagery and the use of mental practice in the development of motor skills. Canadian Journal of Applied Sports Sciences, Vanier, v.10, p.4-6, 1985.

DRISKELL, J.E.; COPPER, C.; MORAN, A. Does mental practice enhance performance? Journal of Applied Psychology, Stanford, v.79, p.481-92, 1994.

EPSTEIN, M.L. The relationship of mental imagery and mental rehearsal to performance of a motor task. Journal of Sport Psychology, Champaign, v.2, p.211-20, 1980.

ETNIER, J.L.; LANDERS, D.M. The influence of procedural variables on the efficacy of mental practice. The Sport Psychologist, Champaign, v.10, p.48-57, 1996.

FELTZ, D.L.; LANDERS, D.M. The effects of mental practice on motor skill learning and performance: a meta-analysis. Journal of Sport Psychology, Champaign, v.5, p.25-57, 1983. 
FONTANI, G.; MIGLIORINI, S.; BENOCCI, R.; FACCHINI, A.; CASINI, M.; CORRADESCHI, F. Effect of mental imagery on the development of skilled motor actions. Perceptual and Motor Skills, Missoula, v.105, p.803-26, 2007. GENTILI, R.; HAN, C.E.; SCHWEIGHOFER, N.; PAPAXANTHIS, C. Motor learning without doing: trial-by-trial improvement in motor performance during mental training. Journal of Neurophysiology, Bethesda, v. 104, p.774-83, 2010. GENTILI, R.; PAPAXANTHIS, C.; POZZO, T. Improvement and generalization of arm motor performance through motor imagery practice. Neuroscience, Amsterdam, v.137, p.761-72, 2006.

GOMES, T.V.B.; BENDA, R.N. Effect of KR supply in mental practice in the aquisition of motor skills. FIEP Bulletin, Foz do Iguaçu, v.75, p.431-3, 2005.

GOMES, T.V.B.; UGRINOWITSCH, H.; BENDA, R.N. Effects of mental practice in novice motor skill acquisition. Journal of Sport and Exercise Psychology, Champaign, v.31, p.S65, 2009.

GOMES, T.V.B.; UGRINOWITSCH, H.; COELHO, R.R.; MARINHO, N.F.S.; FONSECA, F.S.; BENDA, R.N. Efeitos do posicionamento e quantidade de prática mental na aprendizagem do arremesso do dardo de salão. Motriz, Rio Claro, v.18, p.273-9, 2012. GROUIOS, G. The effect of mental practice on diving performance. International Journal of Sport Psychology, Rome, v.23, p.60-9, 1992.

HARRIS, D.V.; ROBINSON, W.J. The effects of skill level on EMG activity during internal and external imagery. Journal of Sport Psychology, Champaign, v.8, p.105-11, 1986.

HIRAI, C.Y.; GOBBI, L.T.B. O desempenho em tarefa motora fina sob diferentes condiçôes de prática. Revista da Educação Física, Maringá, v.2, p.45-50, 1990.

HIRD, J.S.; LANDERS, D.L.; THOMAS, J.R.; HORAN, J.J. Physical practice is superior to mental practice in enhancing cognitive and motor task performance. Journal of Sport \& Exercise Psychology, Champaign, v.8, p.281-93, 1991. MacKAY, D.G. The problem of rehearsal or mental practice. Journal of Motor Behavior, Washington, v.13, p.274-85, 1981. MAGILL, R.A. Motor learning and control: concepts and applications. 8th ed. Boston: McGraw-Hill, 2007.

MARING, J.R. Effects of mental practice on rate of skill acquisition. Physical Therapy, Alexandria, v.70, p.165-72, 1990. McBRJDE, E.R.; ROTHSTEIN, A.L. Mental and physical practice and the learning and retention of open and closed skills. Perceptual and Motor Skills, Missoula, v.49, p.359-65, 1979.

MILLARD, M.; MAHONEY, C.; WARDROP, J. A preliminary study of mental and physical practice on the kayak wet exit skill. Perceptual and Motor Skills, Missoula, v.92, p.977-84, 2001.

MULDER, T.; ZIJLSTRA, S.; ZIJLSTRA, W.; HOCHSTENBACH, J. The role of motor imagery in learning a totally novel movement. Experimental Brain Research, Berlin, v.154, p.211-7, 2004.

NAITO, K.; KATO, T.; FUKUDA, T. Expertise and position of line of sight in golf putting. Perceptual and Motor Skills, Missoula, v.99, p.163-70, 2004.

OVERDORF, V.; SCHWEIGHARDT, R.; PAGE, S.J.; McGRATH, R.E. Mental and physical practice schedules in acquisition and retention of novel timing skills. Perceptual and Motor Skills, Missoula, v.99, p.51-62, 2004.

RAISBECK, L.D.; WYATT, W.R.; SHEA, J.B. A two process memory-based account for mental and physical practice differences. Journal of Motor Behavior, London, v.44, p.115-24, 2012.

RAM, N.; RIGGS, S.M.; SKALING, S.; LANDERS, D.M.; McCULLAGH, P. A comparison of modelling and imagery in the acquisition and retention of motor skills. Journal of Sports Sciences, London, v.25, p.587-97, 2007.

RICHARDSON, A. Mental practice: a review and discussion (Part 1). Research Quartely, Washington, v.38, p.95-107, 1967a. Mental practice: a review and discussion (Part 2). Research Quartely, Washington, v.38, p.263-73, $1967 \mathrm{~b}$.

ROOSINK, M.; ZIJDEWIND, I. Corticospinal excitability during observation and imagery of simple and complex hand tasks: Implications for motor rehabilitation. Behavioural Brain Research, Amsterdam, v.213, p.35-41, 2010.

RYAN, E.D. Experimental error in psychological research: a reaction to "mental practice and knowledge of results in the learning of a perceptual-motor skill”. Journal of Sport Psychology, Champaign, v.5, p.108-10, 1983.

RYAN, E.D.; SIMONS, J. Cognitive demand, imagery, and frequency of mental rehearsal as factors influencing acquisition of motor skills. Journal of Sport Psychology, Champaign, v.3, p.35-45, 1981.

What is learned in mental practice of motor skills: a test of the cognitive-motor hypothesis. Journal of Sport Psychology, Champaign, v.5, p.419-26, 1983.

SCHMIDT, R.A.; LEE, T.D. Motor control and learning. 3rd ed. Champaign: Human Kinetics, 1999.

SHOEKFELT, E.L.; GRIFFITH, M.U. Evaluation of a mental skills program for serving for an intercollegiate volleyball team. Perceptual and Motor Skills, Missoula, v.107, p.293-306, 2008.

SMITH, D.; COLLINS, D. Mental practice, motor performance, and the late CNV. Journal of Sport and Exercise Psychology, Champaign, v.26, p.412-26, 2004. 
STEBBINS, J.R. A comparison of the effects of physical and mental practice in learning a motor skill. Research Quarterly, Washington, v.39, p.714-220, 1968.

SUINN, R. Imagery. In: SINGER, R.N.; MURPHEY, M.; TENNANT, L.K. Handbook of research on sport psychology. New York: MacMillan, 1993. p.492-510.

TANI, G.; MEIRA JUNIOR, C.M.; UGRINOWITSCH, H.; BENDA, R.N.; CHIVIACOWSKY, S.; CORREA, U.C. Pesquisa na área de comportamento motor: modelos teóricos, métodos de investigação, instrumentos de análise, desafios, tendências e perspectivas. Revista da Educação Física, Maringá, v.21, p.329-80, 2010.

TOUSAINT, L.; BLANDIN, Y. On the role of imagery modalities on motor learning. Journal of Sports Sciences, London, v.28, p.497-504, 2010.

TURVEY, M.T. Action and perception at the level of synergies. Human Movement Science, Amsterdam, v.26, p.657-97, 2007. . Preliminaries to a theory of action with reference to vision. In: SHAW, R.E.; BRANSFORD, J. (Eds.). Perceiving, acting and knowing. Hillsdale: Lawrence Erlbaum, 1977. p.211-65.

UGRINOWITSCH, H.; BENDA, R.N. Contribuições da aprendizagem motora: a prática na intervenção em educação física. Revista Brasileira de Educação Física e Esporte, São Paulo, v.25, p.25-35, 2011. Número especial.

VIEIRA, M.M.; UGRINOWITSCH, H.; LAGE, G.M.; BENDA, R.N. Efeitos dos intervalos de tempo de apresentação de conhecimento de resultados (CR) na aquisição de habilidades motoras. Revista Brasileira de Educação Física e Esporte, São Paulo, v.25, p.683-691, 2011.

WEINBERG, R.S. The relationship between mental preparation strategies and motor performance: a review and critique. Quest, Champaign, v.33, p.195-213, 1982.

WEINBERG, R.; HANKES, D.; JACKSON, A. Effect of the length and temporal location of the mental preparation interval on basketball shooting performance. International Journal of Sport Psychology, Rome, v.22, p.3-14, 1991.

WOHLDMANN, E. L.; HEALY, A. F.; BOURNE, L.E. A mental practice superiority effect: less retroactive interference and more transfer than physical practice. Journal of Experimental Psychology: Learning Memory and Cognition, Washington, v.34, p.823-33, 2008.

Pushing the limits of imagination: mental practice for learning sequences. Journal of Experimental Psychology: Learning Memory and Cognition, Washington, v.33, p.254-61, 2007.

YVES, A.F. Differentiating visual and kinesthetic imagery in mental practice. Canadian Journal of Experimental Psychology, Ottawa, v.57, p.1-10, 2003.

ZECKER, S.G. Mental practice and knowledge of results in the learning of a perceptual motor skill. Journal of Sport

Psychology, Champaign, v.4, p.52-63, 1982.

\begin{tabular}{|c|c|}
\hline $\begin{array}{r}\text { ENDEREÇO } \\
\text { Rodolfo Novellino Benda } \\
\text { Escola de Educação Física, Fisioterapia e Terapia Ocupacional } \\
\text { Av. Antônio Carlos, 6627 } \\
\text { 31270-901 - Belo Horizonte- MG - BRASIL } \\
\text { e-mail: rodolfobenda@yahoo.com.br }\end{array}$ & $\begin{array}{l}\text { Recebido para publicação: 16/ 09/2011 } \\
\text { 1a. Revisão: 02/05/2012 } \\
\text { 2a. Revisão: 24/05/2012 } \\
\text { Aceito: 25/ 05/2012 }\end{array}$ \\
\hline
\end{tabular}

